\title{
Quantifying the lagged Poincaré plot geometry of ultrashort heart rate variability series: automatic recognition of odor hedonic tone
}

\author{
M. Nardelli ${ }^{1}$ (D) . G. Valenza ${ }^{1}$ - A. Greco ${ }^{1} \cdot$ A. Lanatá ${ }^{1}$ - E. P. Scilingo ${ }^{1} \cdot$ R. Bailón ${ }^{2,3}$
}

Received: 29 April 2019 / Accepted: 6 December 2019 / Published online: 11 March 2020

(C) International Federation for Medical and Biological Engineering 2020

\begin{abstract}
The application of Poincaré plot analysis to characterize inter-beat interval dynamics has been successfully proposed in the scientific literature for the assessment of humans' physiological states and related aberrations. In this study, we proposed novel descriptors to trace the evolution of Poincaré plot shape over the lags. Their reliability in ultra-short cardiovascular series analysis was validated on synthetic inter-beat series generated through a physiologically plausible integral pulse frequency modulation model. Furthermore, we used the proposed approach for the investigation of the direct relationship between autonomic nervous system (ANS) dynamics and hedonic olfactory elicitation, in a group of 30 healthy subjects. Participants with a similar olfactory threshold were selected, and were asked to score 5-s stimuli in terms of arousal and valence levels according to the Russell's circumflex model of affect. Their ANS response was investigated in 35-s windows after the elicitation. Experimental results showed a gender-specific, high discriminant power of the proposed approach, discerning between pleasant and unpleasant odorants with an accuracy of $83.33 \%$ and $73.33 \%$ for men and for women, respectively.
\end{abstract}

Keywords Lagged poincaré plot $\cdot$ Pattern recognition $\cdot$ Support vector machine $\cdot$ Affective computing $\cdot$ Olfactory elicitation

\section{Introduction}

Many human sensory functions are related to emotional regulation and memory formation, which involve the olfactory system. Previous studies showed that olfaction can be impaired in conditions of chronic stress [1], clinical depression [2], mood disturbances [3], and panic disorder [4]. A decrease in healthy neuronal density of the habenula was recognized in rats presenting depressive symptoms, suggesting that olfactory disorders might be considered one of the possible causes of neuropsychiatric disorders [5]. Odor perception is dominated by smell hedonic tone, and its influence on mood is also due to the anatomical

M. Nardelli

m.nardelli@ing.unipi.it

1 Department of Information Engineering, Research Centre E. Piaggio, University of Pisa, Via G. Caruso 16, Pisa, Italy

2 BSICoS Group, Aragón Institute of Engineering Research (I3A), IIS Aragón, University of Zaragoza, Zaragoza, Spain

3 CIBER of Bioengineering, Biomaterials and Nanomedicine (CIBER - BBN), Madrid, Spain overlap of the olfactory and limbic systems in the brain [6]. Limbic regions are also involved in the regulation of autonomic nervous system (ANS), and significant changes in peripheral physiological dynamics were already reported during olfactory elicitation [7-9].

Heart rate variability (HRV) analysis has emerged as an accessible tool for the objective measure of ANS emotion regulation [10]. Previous literature highlighted an important relationship among HRV, prefrontal neural function, cognitive performances, and emotional response, that has important implications for both mental and physical health [11-13]. Especially in last decades, many algorithms taken from nonlinear theory have been used to study changes in HRV, in relation with pathological moods [1416]. Moreover, several approaches to nonlinear theory have been applied to characterize cardiovascular dynamics during sympatho-vagal unbalancing [17] and to investigate the physiological response during experimental protocols of emotion elicitation using affective images [18, 19], film clips [20, 21], sounds [22, 23], and haptic stimuli [24, 25].

Poincaré plot, or return map, is a geometrical representation of a chaotic system's dynamics. The theoretical background of this approach is Takens' theorem regarding 
the construction of phase space [26]. Poincaré plot analysis can be applied to inter-beat (RR) series derived from the electrocardiogram (ECG), plotting each RR interval against the previous one. Qualitative analysis of Poincaré plot (i.e., visual inspection of its shape) can help in discriminating RR series generated under different cardiovascular diseases [27-29]. Over the past years, several studies have been done with the aim of finding a method to quantify the shape of the Poincaré plot through standard mathematical parameters, regardless the observer's subjectiveness. One of the most used quantitative approaches is the ellipse-fitting method $[30,31]$, which consists in fitting an imaginary ellipse on the scatterplot. Through this method, some quantifiers can be extracted: the area of the ellipse (S), the lengths of the axes (SD1 and SD2), and the ratio between them (SD12). Brennan et al. found the mathematical relationship between SD1 and SD2 and linear parameters extracted from RR series in the time domain [32]. Other studies in the literature demonstrated that ellipse-fitting parameters correlate with other time-domain indexes, e.g., RMSSD and pNN50 [43, 76].

In the lagged Poincare plot (LPP) technique, $R R_{n+M}$ values are plotted against $R R_{n}$ values, being $M$ the lag. Concerning the LPP technique, the ratio between the lengths of the two LPP axes, i.e., SD12, was proposed as an index of the nonlinear structure of the time series [33, 34]. The reliability of LPP ellipse-fitting quantifiers in ultra-short RR series was already investigated using synthetic and real data in our previous studies $[31,35]$.

However, there are several limitations in ellipse-fitting quantifiers, such as the lack of information due to the points not included in the area of the ellipse [36-39]. Concerning the LPP ellipse-fitting, even if we demonstrated its reliability in 35-s series using 10 lags, the percentage absolute errors increase with the lag value. This limitation can be more relevant when a large range of lags is used, e.g., going up to $M=20$, since relevant information might be hidden not only in the shape of Poincare plot but also on the way it evolves with the lag, as we demonstrated in [35, 40].

Here, we propose to augment the information obtained by LPPs using additional quantifiers of the points distribution in the Poincaré plot which take into account all the points in the plot [41], and novel descriptors of the trends of the LPP quantifiers as a function of the lag. These LPP descriptors could allow indexing the trends over the whole range of 20 lags reducing the percentage errors related to ultra-short series at high lags.

First, the reliability of the proposed quantifiers and descriptors of LPP derived from 35-s series is evaluated on synthetic signals generated by means of the integral pulse frequency modulation (IPFM) model. Then, the usefulness of the proposed LPP analysis of HRV in the paradigm of hedonic smell recognition is studied. The aim of the experimental study was to investigate if the ANS response to pleasant and unpleasant odorants can be discerned using only the information inside the heartbeat dynamics. Gender differences in ANS response to olfactory elicitation, already found in a previous study on functional magnetic resonance imaging [42], have been explored in our study.

Paper is organized as follows. First, we describe novel LPP quantifiers and descriptors, and data used in the study (synthetic and real series). Experimental results obtained through statistical analysis and pattern recognition, and Section 4 follows below.

\section{Materials and methods}

\subsection{Lagged Poincaré plot}

\subsubsection{Traditional LPP quantifiers}

Traditional Poincaré plot quantitative analysis is based on the ellipse-fitting method (see Fig. 1) [30]. When this method is applied to LPP, the lagged series $R R_{n+M}$ (where $M$ is the lag) is plotted against $R R_{n}$. The parameters extracted for each lag are the following:

- SD1: the standard deviation of the points calculated along the direction perpendicular to the line-of-identity $R R_{n+M}=R R_{n}$, which describes the short-term dynamics of HRV in the analyzed time interval.

- SD2: the standard deviation of the points along the line-of-identity $R R_{n+M}=R R_{n}$, that describes the long-term variability in HRV dynamics.

- $\operatorname{SD} 12(S D 12=S D 1 / S D 2)$ : the ratio between SD1 and SD2.

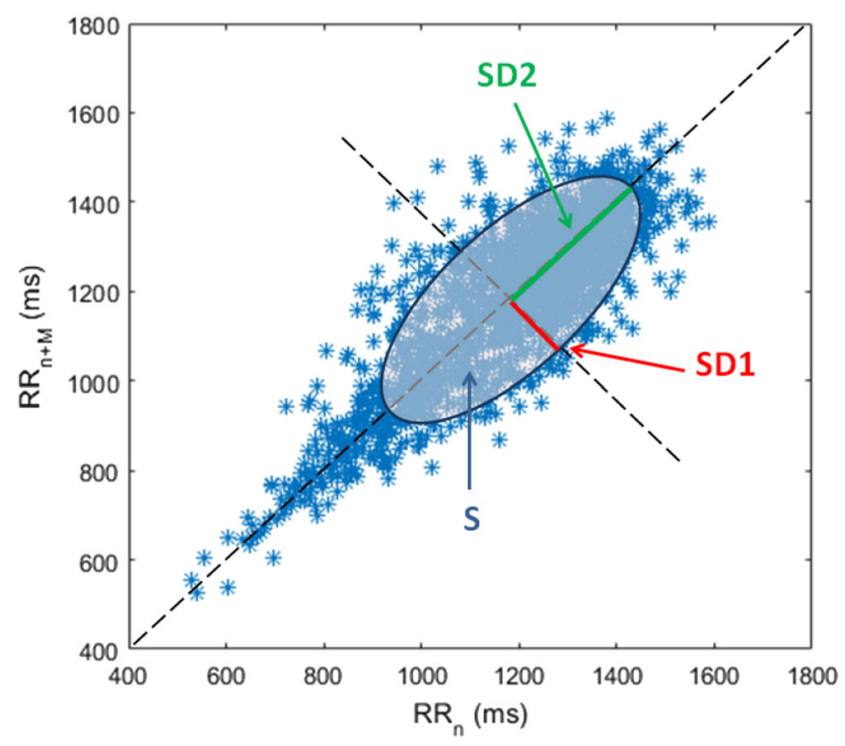

Fig. 1 Scheme of the ellipse-fitting approach to quantify the LPP shape 
- $\mathrm{S}(S=\pi \times S D 1 \times S D 2)$ : the area of an imaginary ellipse with axes SD1 and SD2 [43, 44].

\subsubsection{New LPP approach}

The ellipse-fitting method shows some limitations due to the loss of information when the points lying outside the ellipse are not involved in the quantification. For this reason, we proposed to describe LPP with additional quantifiers which take into account the contribution of all of the points.

For each lag in the LPP method, we identified the position of the centroid $C d$, whose coordinates were calculated as the mean values of the two series used to construct the plot:

$$
\begin{aligned}
C d_{x} & =\frac{1}{N-M} \sum_{n=1}^{N-M} R R_{n} \\
C d_{y} & =\frac{1}{N-M} \sum_{n=M+1}^{N} R R_{n}
\end{aligned}
$$

where $N$ is the total number of $R R$ intervals in the series.

Then, we calculated the geometrical distances $d_{P_{n}}$ between all the points of the Poincaré plot and the centroid, as it is shown in Fig. 2. Afterwards, we computed the mean value and the standard deviation of the distribution of these distances, as described in the following equations:

$$
\begin{aligned}
M_{\mathrm{d}} & =\frac{1}{N-M} \sum_{n=1}^{N-M} d_{P_{n}} \\
S_{\mathrm{d}} & =\sqrt{\frac{\sum_{n=1}^{N-M}\left(d_{P_{n}}-M_{\mathrm{d}}\right)^{2}}{N-M-1}} .
\end{aligned}
$$

For each series, we considered 20 LPPs $(1 \leq M \leq$ 20). In order to evaluate the changes in the trends of the LPP quantifiers as a function of $M$, we used the following descriptors: (i) the values of the quantifiers at the first lag ( $M=1$, the traditional descriptor), (ii) the maximum values of each quantifier along the lags, and iii) the area under the

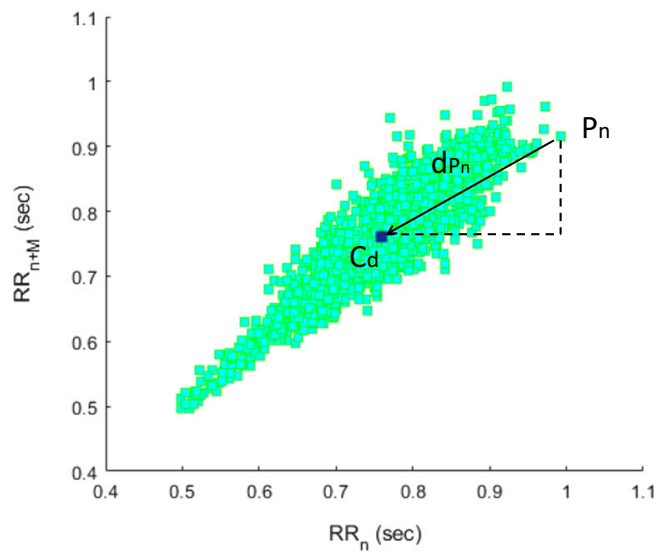

Fig. 2 Scheme of the new approach to quantify the LPP shape. $C d\left(C d_{x}, C d_{y}\right)$ indicates the centroid (in blue) and $d_{P_{n}}$ is the distance between one point, $P_{n}$, and the centroid curve described by the LPP quantifiers, as a function of the lag $M$. We employed the following notation for these novel descriptors (for example, considering $\left.M_{\mathrm{d}}\right): \max \left(M_{\mathrm{d}}\right)$ for the maximum, and $\operatorname{auc}\left(M_{\mathrm{d}}\right)$ for the area under the $M_{\mathrm{d}}$ curve.

\subsection{Data used in the study}

In this study, we used two datasets, the first of synthetic RR series and the second of real data (see Sections 2.2.1 and 2.2.2). Synthetic series were used to test the reliability of new LPP quantifiers and descriptors in ultra-short RR series. The experimental dataset consisted in the ECG signals recorded during a protocol of olfactory elicitation.

\subsubsection{Synthetic series}

We generated 1200 synthetic RR series through the timeinvariant version of IPFM model [45]. This model simulates the heartbeat occurrence times from a modulating signal $m(t)$, which describes the oscillations of ANS drive.

The $k$ th beat trigger impulse is generated when the integral of $1+m(t)$, reaches a threshold $T$, which represents the mean RR:

$k=\int_{0}^{t_{k}} \frac{1+m(t)}{T} d t$

where $k$ is an integer that represents the $k$ th beat and $t_{k}$ is the occurrence time of the $k$ th beat. Modulating signal $m(t)$ is realized by means of an autoregressive moving average model, using both the frequency of the dominant peak and the power in the LF and HF bands as input parameters [46]. In this study, the frequencies of the peaks were randomly chosen in the range of the two main bands (LF and HF) and their power values were within the normal range for healthy people reported in literature [47, 48]. Half of the series were generated fixing a value of LF power and varying HF power (20 different values). For each couple of frequency parameters (LF power and HF power), 30 series were generated. Then, the process is repeated keeping HF power constant and varying LF power. The parameter $\mathrm{T}$ was set to $1 \mathrm{~s}$ for all the realizations. A total amount of 1200 realizations of the modulating signal $m(t)$ were generated. The duration of each RR series was of $1 \mathrm{~h}$.

\subsubsection{Experimental data acquired during olfactory elicitation}

Thirty-two participants aged $26 \pm 2$ (16 females) took part in the study. They gave their informed consent before the experiment and self-reported no history of clinical and sub-clinical cardiovascular diseases. No participants reported physical limitations or any experience of mental or personality disorder in their life that would affect the 
experimental outcomes. Before starting the experimental procedure, participants were asked to fill out some questionnaires to evaluate the level of subjectively perceived anxiety (STAI-Y, [74] and the mood (PANAS, [75]. Participants whose scores were not within the normative ranges of PANAS and STAI-Y (e.g., STAI-Y scores > 45, and PANAS: Negative affect NA $>30$ e Positive affect $\mathrm{PA}<18$ ) were not included in the study, thus indicating the possible presence of affective disorders. This study was approved by the Ethical Committee of the University of Pisa.

During the experimental protocol, the participants were comfortably seated and they wore earplugs in order to prevent any auditory cues.

Two odorants, normally considered pleasant and unpleasant, already reported in previous studies in the literature [49], were used in the experimental protocol:

- $\mathrm{Od}_{1}$ : Benzaldehyde $\mathrm{C}_{6} \mathrm{H}_{5} \mathrm{CHO}$, concentration 106,12 $\mathrm{g} / \mathrm{mol}$;

- $\mathrm{Od}_{2}$ : Isovaleric acid $\left(\mathrm{CH}_{3}\right)_{2} \mathrm{CHCH}_{2} \mathrm{COOH}$, concentration $102,13 \mathrm{~g} / \mathrm{mol}$.

Benzaldehyde odor is a pleasant bitter almond smell, whereas isovaleric acid smells like an unpleasant sweat odor. The concentrations of the two odorants were selected in order to obtain stimuli of a comparable intensity. We recruited a group of volunteers exhibiting a similar olfactory threshold to N-butanol $\left(\mathrm{CH}_{3} \mathrm{CH}_{2} \mathrm{CH}_{2} \mathrm{CH}_{2} \mathrm{OH}, 74.12 \mathrm{~g} / \mathrm{mol}\right)$ [50]. This odorant is suggested from the literature as the most suitable neutral smell, in order to identify the olfactory threshold level. The procedure of selection was as follows:

- Ten solutions of N-butanol at different concentration were prepared, by diluting a mother solution $(4.05 \mathrm{~g} / \mathrm{L})$ with dilution factor chosen as powers of two, from 8 to 4096.

- The subject was asked to recognize N-butanol solution from distilled water; we started from the lowest concentration, increasing until the subject was able to clearly discern the odorant.
- If the dilution factor of the solution recognized was in the range 256-1024, the subject was selected for the experiment.

During the experiment, the order of the stimuli was randomized and after each olfactory stimulus, the participants were asked to assess their sensation with two numbers, in terms of arousal (i.e., the intensity of the evoked emotion, ranged from 1 to 5) and valence (i.e., pleasantness/unpleasantness of the stimulus, ranged from -2 to +2 ), following the bidimensional circumplex model of affect (CMA) of Russell [51]. In order to help the subjects to express the values of arousal and valence, we used the Self Assessment Manikin (SAM), a graphical interpretation of Russell's model [52]. This self assessment was used to verify that the two odorants chosen for the experimental protocol had effectively stimulated the emotion response of subjects. The two dimensions of CMA allowed us to understand if the two odorants have been perceived with the same intensity (arousal) and opposed levels of valence, as we expected in the design of the experimental protocol. The timeline of the whole experiment is displayed in Fig. 3. Two resting state sessions of 3 min were at the beginning and at the end. The two olfactory stimuli lasted $5 \mathrm{~s}$ and were presented between two 1-min rest periods (pre-stimulus and post-stimulus rest sessions). The self assessment with SAM was after the post-stimulus rest and lasted $20 \mathrm{~s}$.

The ECG was continuously acquired following the Einthoven triangle configuration, using the ECG100C Electrocardiogram Amplifier from BIOPAC inc. with a sampling rate of $500 \mathrm{~Hz}$. The RR series were extracted from ECG signals, using a QRS complex detection algorithm, i.e., the automatic algorithm developed by Pan-Tompkins [53]. Artifacts and ectopic beats were corrected through the use of Kubios HRV software [54].

\subsection{HRV feature extraction}

LPP quantifiers (traditional and new) have been extracted from 1200 synthetic series. We calculated the quantifiers

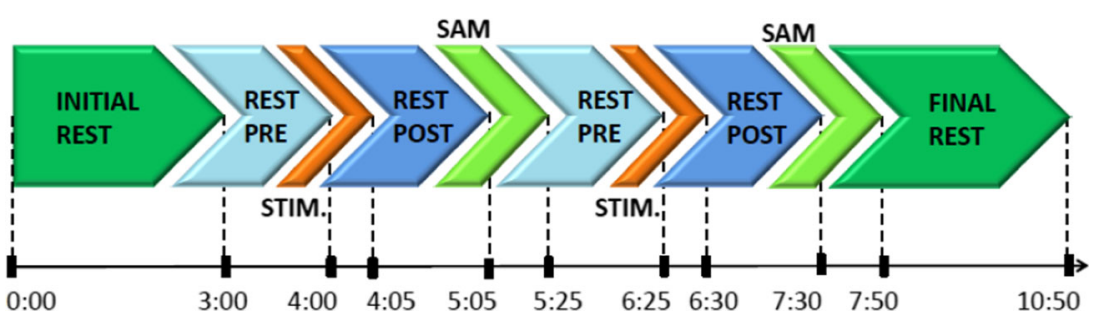

Fig. 3 Timeline of the experimental protocol for olfactory stimulation. REST PRE, resting state session before olfactory elicitation; STIM, olfactory stimulus presentation; REST POST, resting state session after olfactory elicitation; SAM, Self Assessment Manikin test 
described in Section 2.1 for all of the lags from 1 to 20, then we extracted the values of the auc and max from each LPP trend as a function of M lag. For each LPP quantifier (SD1, $\mathrm{SD} 2, \mathrm{SD} 12, S, M_{\mathrm{d}}$, and $S_{\mathrm{d}}$ ), three descriptor values were studied: the value at the first lag $(M=1)$, the value of auc, and max (for lags from 1 to 20).

The RR synthetic series have been analyzed in 50\% overlapped windows of $35 \mathrm{~s}$. The LPP parameters' value was estimated as the median in all the windows of the same length and considering the total length of synthetic series (1 hour), in order to test their reliability.

RR series acquired during the olfactory stimulation were analyzed during the pre-stimulus and post-stimulus rest stimulus lasting $1 \mathrm{~min}$. As in the processing of synthetic RR series, we computed the HRV analysis considering 50\% overlapped windows of $35 \mathrm{~s}$, two windows for each 1min experimental session. In order to investigate if LPP technique adds relevant information to linear HRV indices (time and frequency domain), these later parameters were also estimated. We calculated two parameters in the time domain: the mean value of the durations of all RR intervals (RR mean) and their standard deviation (RR std).

The smoothness priors approach was applied to each RR series as detrending method [73], operating a timevarying finite-impulse response high-pass filter with a cutoff frequency of $0.035 \mathrm{~Hz}$. The HRV signals were derived by applying the IPFM model, which also accounts for the presence of ectopic beats [55], and then resampled at $4 \mathrm{~Hz}$, using spline interpolation. Then, we estimated the power spectral density (PSD) in each 35-s rectangular window, with a $50 \%$ overlap. From the PSD related to each time window, we extracted six features. We computed the power within the low frequency (LF), from 0.04 to $0.15 \mathrm{~Hz}$ and the high frequency (HF), from 0.15 to $0.4 \mathrm{~Hz}$ (LF power and HF power). The ratio between LF power and $\mathrm{HF}$ power $(\mathrm{LF} / \mathrm{HF})$, the power in $\mathrm{HF}$ band normalized to the sum of LF and HF power (HFn), and the values of the frequencies having maximum magnitude (LF peak and HF peak) were also calculated. Time and frequency features and LPP descriptors and quantifiers were extracted from $35 \mathrm{~s}$ windows and then the median of the two periods in each 1-min resting session was computed.

\subsection{Statistical analysis and pattern recognition}

\subsubsection{Reliability of LPP parameters estimated 35 second series}

To test the reliability of novel LPP approach, we considered the percentage absolute error $(\epsilon \%)$ between the median value of the parameters calculated in all the windows of $35 \mathrm{~s}$ and the value of the parameter calculated on the total length of synthetic series $(1 \mathrm{~h})[31,35]$. The median and median absolute deviation (MAD) of the $\epsilon \%$ values obtained for all the 1200 synthetic RR series is then computed.

\subsubsection{Discrimination of olfactory stimuli using LPP parameters}

To analyze real RR series in terms of statistical analysis and pattern recognition, we considered the median values of the LPP descriptors belonging to the two 35-s windows in each pre- and post-stimuli session. Then, we subtracted the values of the the pre-stimuli sessions to the values calculated in the post-stimuli sessions, in order to check the impact of olfactory elicitation on ANS dynamics. Given the nonGaussianity of data distributions, we applied non-parametric Wilcoxon statistical test [56] between the values of post-pre differences calculated for the two different stimuli.

The main aim was to test if the new descriptors (auc and max), calculated from ellipse-fitting LPP quantifiers and $M_{\mathrm{d}}$ and $S_{\mathrm{d}}$ quantifiers, were able to discern the differences between the physiological responses to hedonic olfactory stimuli and to study the added value with respect to the classical Poincaré plot quantifiers. To reach this objective, we implemented a Leave-One-Subject-Out (LOSO) procedure [57] and a feature selection was performed by means of a recursive feature elimination based on Support Vector Machine (SVM-RFE) [58]. Then, we applied a C-Support Vector Classifier (C-SVC) with a radial basis function kernel.

We applied the statistical and classification procedure in three scenarios: all the subjects together, only men, and

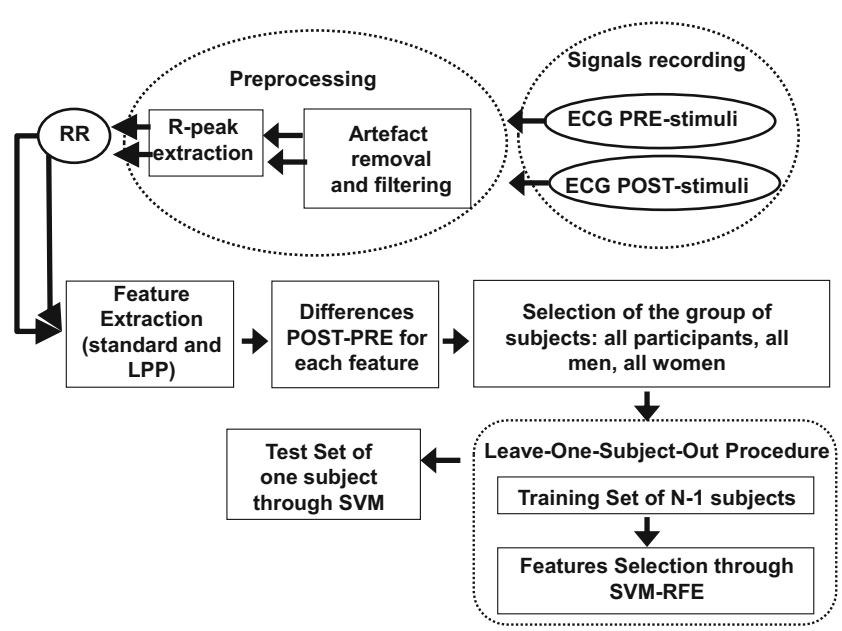

Fig. 4 Block scheme of data analysis. Data acquisition (ECG signals in pre and post-stimuli sessions), signal pre-processing (application of Pan-Tompkins algorithm and artifact removal using Kubios HRV software), feature extraction (time and frequency domains and LPP), computation of differences between feature values in pre e and post-stimuli sessions and machine learning procedure performed to automatically characterize the hedonic olfactory stimulation 
Table 1 Values of $\epsilon \%$ for LPP descriptors extracted from 35-s windows with respect to those extracted from $1 \mathrm{~h}$, among the 1200 synthetic series

\begin{tabular}{lll}
\hline & auc & $\max$ \\
\hline $\mathrm{SD} 1$ & $8.14 \pm 1.59$ & $7.33 \pm 2.16$ \\
$\mathrm{SD} 2$ & $8.75 \pm 1.77$ & $7.46 \pm 2.15$ \\
$S$ & $17.38 \pm 3.06$ & $14.42 \pm 3.56$ \\
$\mathrm{SD} 12$ & $0.86 \pm 0.54$ & $2.69 \pm 1.65$ \\
$M_{\mathrm{d}}$ & $5.06 \pm 1.78$ & $4.19 \pm 1.79$ \\
$S_{\mathrm{d}}$ & $23.50 \pm 3.20$ & $18.47 \pm 2.68$ \\
\hline
\end{tabular}

The errors are presented as median \pm MAD

only women. In the statistical analysis and classification procedure, we considered all the subjects with an RR mean not higher than one second to compute the LPP parameters at the 20th lag using at least 10 samples. For this reason, two subjects (one man and one woman) were discarded from the analyses. The overall scheme of the whole procedure of processing of the ECG signals acquired during the olfactory stimulation protocol is shown in Fig. 4. The results of the pattern recognition algorithm are reported in this study as percentage values of sensitivity, specificity, and accuracy (Table 4) related to the best performances reached with the three datasets.

\section{Experimental results}

\subsection{Synthetic series}

The median percentage absolute errors $(\epsilon \%)$ extracted from the 1200 series are displayed in Table 1. They
Fig. 5 Boxplots of arousal (left column) and valence (right column) values correspondent to the two smells for the three datasets. Whole subjects $(\mathbf{a}, \mathbf{b})$, male subjects $(\mathbf{c}, \mathbf{d}$,$) and female$ subjects $(\mathbf{e}, \mathbf{f})$

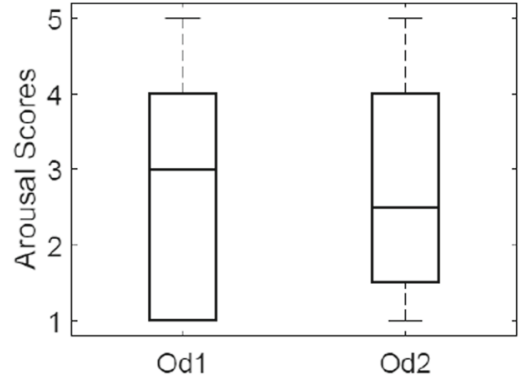

(a) All subjects (AR)

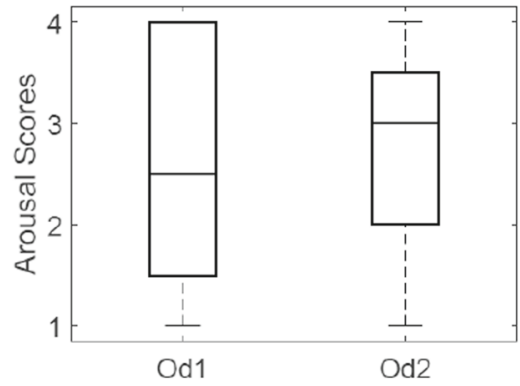

(c) Men (AR)

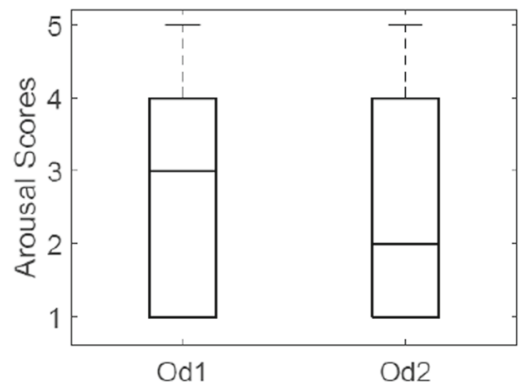

(e) Women (AR)

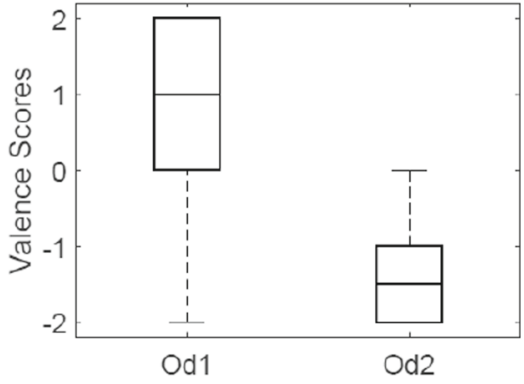

(b) All subjects (VAL)

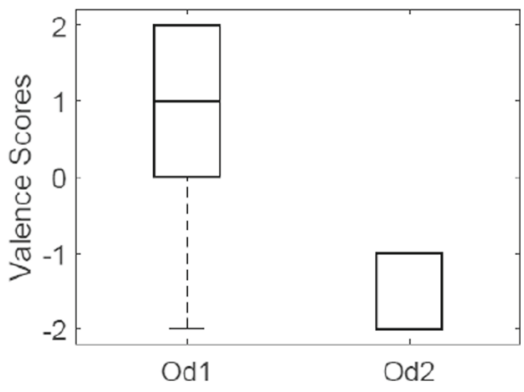

(d) Men (VAL)

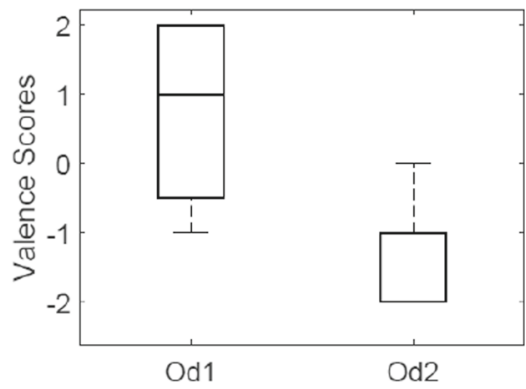

(f) Women (VAL) 
were computed comparing the median value of novel LPP descriptors and quantifiers in windows of $35 \mathrm{~s}$ with the same parameters extracted from total length of synthetic series, 1 h. $\epsilon \%$ was calculated for the novel descriptors, i.e., auc and max. Even if the novel descriptors, auc and max, took into account 20 values of lag, their errors remained comparable with $\epsilon \%$ obtained from the value of the same quantifiers considering the first lag only (previously shown in [31]). SD12 and $M_{\mathrm{d}}$ resulted the most reliable quantifiers for all the descriptors: the median value of $\epsilon \%$ was less than $0.9 \%$ and $2.7 \%$ when it was computed on auc(SD12) and max(SD12), and it was less than $5.1 \%$ and $4.2 \%$ when it was computed on $\operatorname{auc}\left(M_{\mathrm{d}}\right)$ and $\max \left(M_{\mathrm{d}}\right)$, respectively.

\subsection{Experimental data during olfactory stimulation}

\subsubsection{Statistical results of SAM}

For both arousal and valence dimensions of Russell's model, and for each group taken into account in our study (all subject, only men, only women), we applied the Wilcoxon statistical test to compare the two sub-samples of SAM scores related to pleasant and unpleasant olfactory stimuli, respectively.

No significant differences were found among the arousal scores in the three groups (all subjects, all men, all women) (see Fig. 5). When we applied Wilcoxon test to the valence dimension, we found that valence scores were significantly different in the three groups of participants $\left(p<10^{-6}\right), \mathrm{Od}_{1}$ was identified as pleasant odorant, whereas $\mathrm{Od}_{2}$ was scored with a negative valence.

\subsubsection{Statistical results of HRV parameters}

Wilcoxon non-parametric statistical tests were applied to the post-pre values of time and frequency parameters and LPP parameters extracted from 35-s RR series, in order to discern the features able to distinguish the stimuli. In Table 2, the statistical results are reported in terms of $p$ values, for the three following groups: all 30 subjects, all men ( 15 subjects), and all women (15 subjects). The lowest $p$ values considering all the subjects were obtained for $M_{\mathrm{d}(M=1)}$ and $(\max ) M_{\mathrm{d}}$. As regards the men's group, the most significant features were for $M_{\mathrm{d}}(M=1)$ and LF power. In the group of women, the only significant feature was RR mean. The boxplots of the two most significant features in the groups of all participants and all men are displayed in Figs. 6 and 7. Figure 8 shows the boxplot related to RR mean for the group of women. All the significant features showed the same trend in the median values: a decrease during the post-stimuli session after the pleasant smell and an increase after the unpleasant stimulation (see Table 2).

It is worthwhile pointing out that 14 out of 17 features which gave significant results in the statistical analysis,

Table 2 Wilcoxon statistical tests between the values of features during pleasant $\left(\mathrm{Od}_{1}\right)$ and unpleasant $\left(\mathrm{Od}_{2}\right)$ olfactory stimulation

\begin{tabular}{|c|c|c|c|}
\hline Parameter & $p$ val & Od 1 & Od2 \\
\hline & \multicolumn{3}{|c|}{ All subjects } \\
\hline$M_{\mathrm{d}}(M=1)$ & 0.0028 & - & + \\
\hline $\max M_{\mathrm{d}}$ & 0.0030 & - & + \\
\hline LFpower & 0.0034 & - & + \\
\hline $\operatorname{auc}\left(M_{\mathrm{d}}\right)$ & 0.0036 & - & + \\
\hline $\mathrm{SD} 2(M=1)$ & 0.0036 & - & + \\
\hline $\max (\mathrm{SD} 2)$ & 0.0041 & - & + \\
\hline auc(SD2) & 0.0064 & - & + \\
\hline $\max (S)$ & 0.0077 & - & + \\
\hline $\max (\mathrm{SD} 1)$ & 0.0087 & - & + \\
\hline $\operatorname{auc}(S)$ & 0.0093 & - & + \\
\hline $\operatorname{auc}(\mathrm{SD} 1)$ & 0.0117 & - & + \\
\hline $\mathrm{S}(M=1)$ & 0.0117 & - & + \\
\hline$S_{\mathrm{d}}(M=1)$ & 0.0175 & - & + \\
\hline $\max \left(S_{\mathrm{d}}\right)$ & 0.0196 & - & + \\
\hline RRstd & 0.0256 & - & + \\
\hline $\operatorname{auc}\left(S_{\mathrm{d}}\right)$ & 0.0285 & - & + \\
\hline \multirow[t]{2}{*}{ HFpower } & 0.0495 & - & + \\
\hline & \multicolumn{3}{|l|}{ Men } \\
\hline$M_{\mathrm{d}}(M=1)$ & 0.0084 & - & + \\
\hline LFpower & 0.0125 & - & + \\
\hline $\max (\mathrm{SD} 1)$ & 0.0151 & - & + \\
\hline $\operatorname{auc}\left(M_{\mathrm{d}}\right)$ & 0.0151 & - & + \\
\hline $\max (\mathrm{SD} 2)$ & 0.0181 & - & + \\
\hline auc(SD12) & 0.0181 & - & + \\
\hline $\max \left(M_{\mathrm{d}}\right)$ & 0.0181 & - & + \\
\hline $\mathrm{SD} 2(M=1)$ & 0.0181 & - & + \\
\hline $\max (S)$ & 0.0215 & - & + \\
\hline auc(SD1) & 0.0302 & - & + \\
\hline $\operatorname{auc}(S)$ & 0.0302 & - & + \\
\hline $\max (\mathrm{SD} 12)$ & 0.0413 & - & + \\
\hline \multirow[t]{2}{*}{ auc(SD2) } & 0.0479 & - & + \\
\hline & \multicolumn{3}{|c|}{ Women } \\
\hline RRmean & 0.0353 & - & + \\
\hline
\end{tabular}

For the two groups of subjects (all participants, all men), the $p$ value of the test is shown. Symbols + and - refer to the signs of the differences post - pre in the parameters' values 

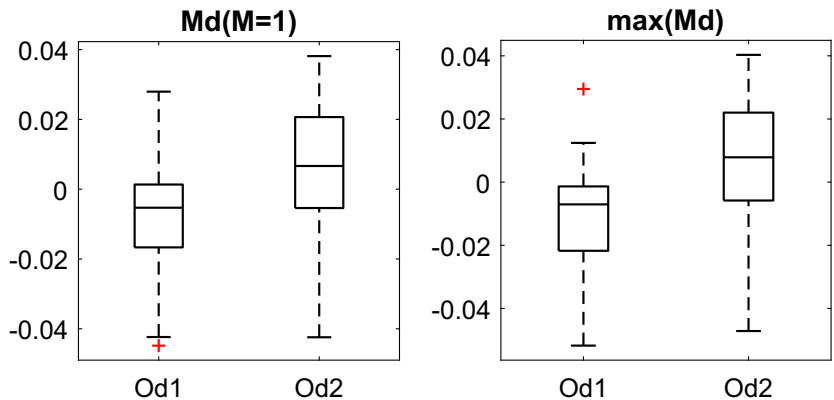

Fig. 6 Boxplots of parameters $M_{\mathrm{d}}(M=1)$ and $\max \left(M_{\mathrm{d}}\right)$ for all the subjects. Values refer to the differences post-pre for the two different odorants $\mathrm{Od}_{1}$ and $\mathrm{Od}_{2}$

considering all the subjects together, were extracted from the LPP approach proposed in this study.

In Table 3, we reported the median and MAD computed in both pre- and post-stimulus sessions, for each parameter used in the study and for both odorants.

\subsubsection{Pattern recognition}

We used the differences post-pre, calculated for all the features described, as input to a LOSO procedure in order to classify the two olfactory stimuli. In Fig. 9, the accuracy trends, as a function of the number of features considered as input of the pattern recognition algorithm, are presented for both male and female groups.

The best results in terms of accuracy in the classification of the two smells are shown in Table 4, for each group (all subjects, men, and women). Figure 9 and Table 4 are related to pattern recognition results obtained when all the features (time and frequency domains, and LPP descriptors) are used as input of pattern recognition algorithm. Considering all the subjects, the maximum level of accuracy was $71.67 \%$ and was reached using one feature (auc(SD2) (see Table 4). In the results related to men, the maximum value achieved with the proposed algorithm was $83.33 \%$ with three
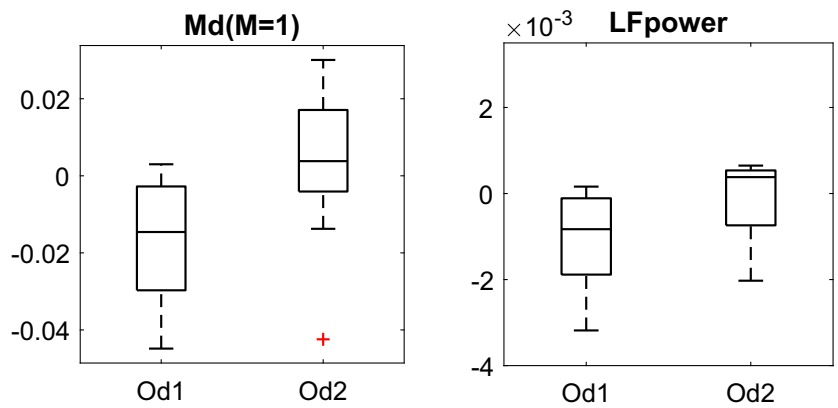

Fig. 7 Boxplots of parameters $M_{\mathrm{d}}(M=1)$ and LF power for the group of men. Values refer to the differences post-pre for the two different odorants $\mathrm{Od}_{1}$ and $\mathrm{Od}_{2}$

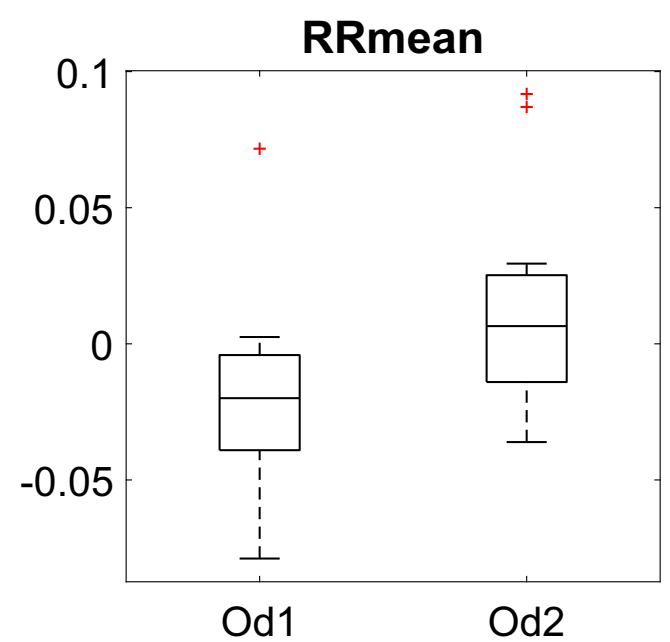

Fig. 8 Boxplots of RR mean, the only statistically significant parameter, for the group of women. Values refer to the differences post-pre for the two different odorants $\mathrm{Od}_{1}$ and $\mathrm{Od}_{2}$

features: $\operatorname{auc}\left(S_{\mathrm{d}}\right), \max \left(S_{\mathrm{d}}\right)$, and $\mathrm{HFn}$. We found a maximum of accuracy of $73.33 \%$ with three features $(S(M=1)$, $M_{\mathrm{d}}(M=1)$ and HF power) when we restricted the analysis to data recorded only from women (Table 4). As it can be easily noticed, all the most frequently selected features belonged to the novel LPP quantifiers and descriptors here proposed.

Table 5 shows the comparison between the results obtained with the pattern recognition algorithm using different feature subsets. We considered the following input subsets: time-domain HRV features, frequency-domain HRV features, and LPP descriptors. The algorithm of pattern recognition was computed considering the three groups (all subjects, only men, and only women), for the three feature subsets of HRV features. From the results reported in Table 5, we noticed that using both time and frequency domain datasets the maximum of accuracy did not reach values upper than $70 \%$. For both the groups of all subjects and all men, the highest percentage of accuracy was obtained using the LPP parameter subset $(70.00 \%$ and $76.67 \%$, respectively), whereas for the female group, we reached the maximum of accuracy using RR mean $(73.33 \%)$.

\section{Discussion}

In this study, we presented an ad hoc algorithm for pattern recognition able to recognize different $\mathrm{HRV}$ responses to two odorants, one pleasant and one unpleasant. A novel approach to describe the trend of LPPs obtained from ultra-short RR series is described and validated, as an efficient complementary method that can produce 
Table 3 Median and MAD values related to each parameter in the pre and post-stimulus session for both odorants (Od1 and Od2)

\begin{tabular}{|c|c|c|c|c|c|c|c|c|}
\hline \multirow[t]{3}{*}{ Parameter } & \multicolumn{4}{|l|}{ Od1 } & \multicolumn{4}{|l|}{$\mathrm{Od} 2$} \\
\hline & \multicolumn{2}{|l|}{ Pre- } & \multicolumn{2}{|l|}{ Post- } & \multicolumn{2}{|l|}{ Pre- } & \multicolumn{2}{|l|}{ Post- } \\
\hline & Median & MAD & Median & MAD & Median & MAD & Median & MAD \\
\hline RRmean & 0.8250 & 0.1096 & 0.8243 & 0.0944 & 0.8136 & 0.0941 & 0.7923 & 0.0978 \\
\hline RRstd & 0.0477 & 0.0163 & 0.0465 & 0.0141 & 0.0401 & 0.0098 & 0.0490 & 0.0093 \\
\hline LF peak & 0.0743 & 0.0145 & 0.0764 & 0.0179 & 0.0911 & 0.0290 & 0.0886 & 0.0214 \\
\hline HF peak & 0.2106 & 0.0333 & 0.2206 & 0.0448 & 0.2076 & 0.0318 & 0.1970 & 0.0331 \\
\hline LF power & 0.0014 & 0.0009 & 0.0012 & 0.0006 & 0.0009 & 0.0005 & 0.0010 & 0.0005 \\
\hline HF power & 0.0009 & 0.0005 & 0.0008 & 0.0003 & 0.0006 & 0.0004 & 0.0006 & 0.0004 \\
\hline HFn & 0.3626 & 0.1646 & 0.3617 & 0.1575 & 0.4405 & 0.1358 & 0.3410 & 0.1182 \\
\hline $\mathrm{LF} / \mathrm{HF}$ & 1.9241 & 1.2505 & 2.2594 & 1.4919 & 1.2808 & 0.6497 & 2.0248 & 1.3648 \\
\hline $\operatorname{auc}(\mathrm{SD} 1)$ & 0.8548 & 0.2110 & 0.7868 & 0.2422 & 0.7343 & 0.2078 & 0.8474 & 0.1407 \\
\hline auc(SD2) & 0.8814 & 0.2709 & 0.7776 & 0.2282 & 0.7419 & 0.1533 & 0.8730 & 0.1454 \\
\hline $\operatorname{auc}(S)$ & 0.1213 & 0.0617 & 0.0978 & 0.0607 & 0.0941 & 0.0435 & 0.1204 & 0.0355 \\
\hline $\max (\mathrm{SD} 1)$ & 0.0568 & 0.0157 & 0.0521 & 0.0146 & 0.0499 & 0.0139 & 0.0550 & 0.0097 \\
\hline $\max (\mathrm{SD} 2)$ & 0.0633 & 0.0189 & 0.0564 & 0.0162 & 0.0541 & 0.0115 & 0.0623 & 0.0111 \\
\hline $\max (S)$ & 0.0075 & 0.0042 & 0.0059 & 0.0037 & 0.0056 & 0.0026 & 0.0073 & 0.0022 \\
\hline $\operatorname{auc}\left(M_{\mathrm{d}}\right)$ & 1.1321 & 0.2865 & 0.9772 & 0.2952 & 0.9686 & 0.2284 & 1.1193 & 0.1678 \\
\hline $\operatorname{auc}\left(S_{\mathrm{d}}\right)$ & 0.5715 & 0.1360 & 0.5276 & 0.1362 & 0.4451 & 0.1216 & 0.5198 & 0.1016 \\
\hline auc(SD12) & 20.2631 & 1.4814 & 19.8233 & 1.0470 & 19.6755 & 1.2315 & 20.3251 & 0.8403 \\
\hline $\max (\mathrm{SD} 12)$ & 1.7313 & 0.2870 & 1.7265 & 0.2338 & 1.6315 & 0.1743 & 1.8039 & 0.2003 \\
\hline $\max \left(M_{\mathrm{d}}\right)$ & 0.0623 & 0.0186 & 0.0559 & 0.0161 & 0.0536 & 0.0125 & 0.0628 & 0.0097 \\
\hline $\max \left(S_{\mathrm{d}}\right)$ & 0.0351 & 0.0112 & 0.0353 & 0.0095 & 0.0280 & 0.0074 & 0.0320 & 0.0063 \\
\hline $\operatorname{SD} 1(M=1)$ & 0.0243 & 0.0058 & 0.0225 & 0.0072 & 0.0207 & 0.0056 & 0.0219 & 0.0058 \\
\hline $\mathrm{SD} 2(M=1)$ & 0.0633 & 0.0204 & 0.0563 & 0.0159 & 0.0537 & 0.0113 & 0.0608 & 0.0121 \\
\hline $\operatorname{SD} 12(M=1)$ & 0.3848 & 0.0607 & 0.4115 & 0.0922 & 0.4456 & 0.0973 & 0.3971 & 0.0875 \\
\hline $\mathrm{S}(M=1)$ & 0.0050 & 0.0022 & 0.0045 & 0.0017 & 0.0036 & 0.0017 & 0.0041 & 0.0021 \\
\hline$M_{\mathrm{d}}(M=1)$ & 0.0590 & 0.0145 & 0.0520 & 0.0114 & 0.0484 & 0.0109 & 0.0569 & 0.0088 \\
\hline$S_{\mathrm{d}}(M=1)$ & 0.0348 & 0.0112 & 0.0349 & 0.0097 & 0.0265 & 0.0078 & 0.0315 & 0.0057 \\
\hline
\end{tabular}

Values are reported in seconds, except for LF peak and HF peak reported in $\mathrm{Hz}$, and LF power and HF power reported in $\mathrm{s}^{2}$

emotion-discriminant features. Specifically, we used 35-s series to test our approach, in order to be easily implemented in e-health and mobile applications using ultra-short cardiovascular series, with a low computational cost.

Nonlinear methods were demonstrated to have a strong discriminative power in the recognition of emotional states $[14,15,59]$ and affective response to external stimuli [22, 31]. However, one of the most important limitations of nonlinear analysis, when it is applied to HRV series, is that most nonlinear metrics present better performances in the study of long-term monitoring acquisitions and result to be less reliable than linear parameters in ultra-short series analysis [60].

In our previous works, we studied the reliability of LPP ellipse-fitting quantifiers in ultra-short RR series [35, 61], using 1200 synthetic series generated through the IPFM model [45]. Even if we showed that ellipse-fitting technique

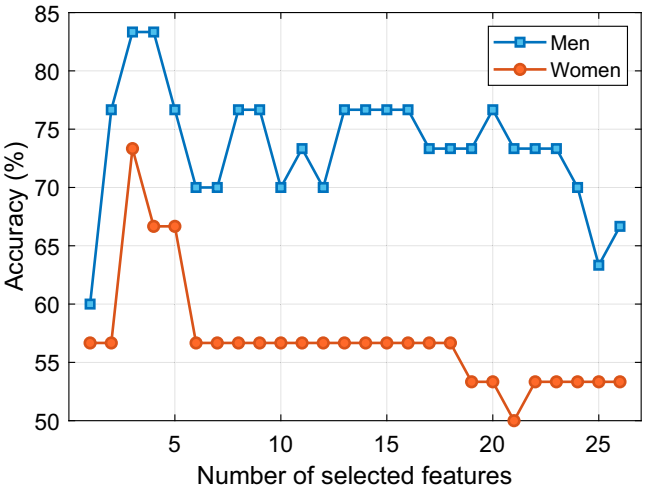

Fig. 9 Recognition accuracy for $\mathrm{Od}_{1}$ vs. $\mathrm{Od}_{2}$ classification obtained through the LOSO SVM-RFE procedure, shown as a function of the feature number, according to the gender 
Table 4 Best results obtained for the classification of $\mathrm{Od}_{1}$ and $\mathrm{Od}_{2}$ through SVM, for the three groups in the study (all subjects, all men, and all women)

\begin{tabular}{llll}
\hline & All subjects & Men & Women \\
\hline Most frequently & $\operatorname{auc}(\mathrm{SD} 2)$ & $\operatorname{auc}\left(S_{\mathrm{d}}\right)$ & $S(M=1)$ \\
selected features & & $\max \left(S_{\mathrm{d}}\right)$ & $M_{\mathrm{d}}(M=1)$ \\
& & $\mathrm{HFn}$ & HFpower \\
Sensitivity & $70.00 \%$ & $100.00 \%$ & $86.67 \%$ \\
Specificity & $73.33 \%$ & $66.67 \%$ & $60.00 \%$ \\
Accuracy & $71.67 \%$ & $83.33 \%$ & $73.33 \%$ \\
\hline
\end{tabular}

For each dataset, the most frequently features selected by RFE and the values of sensitivity, specificity, and accuracy are reported produces low percentage errors in series lasting less than $1 \mathrm{~min}$, this error increased according to the lag [31]. This rise was even more evident in the case of SD12, which is also the LPP quantifiers derived from ellipse-fitting theory associated with nonlinear cardiac dynamics [34]. The new LPP descriptors proposed here resulted to be reliable for ultra-short series also considering 20 lags, as it is shown in Table 1, through the results of percentage absolute error between the values of LPP descriptors calculated in 35-s segments, compared with 1-h synthetic series. As we can see in Table 1, the median value of percentage absolute error was always less than $5.1 \%$ and $4.2 \%$, when $\operatorname{auc}\left(M_{\mathrm{d}}\right)$ and $\max \left(M_{\mathrm{d}}\right)$ were considered.

Following these findings, we applied the proposed approach in the analysis of ultra-short RR series, recorded during a protocol of olfactory stimulation. The physiological expression of emotions in response to odorants was analyzed in several previous studies in the literature [49, 6264], and a strict link between odors perception and limbic system activity was found, specifically amygdala and hippocampus $[65,66]$. These areas are known to be involved in the control of crucial homeostatic functions in response to external stimuli and especially the amygdala receives interoceptive afferents and may play a role in ANS functions $[66,67]$. The effect of fragrances on ANS was discussed by Van Toller, who recognized the power of the primitive sense of smell measuring ANS response through the study of electrodermal activity [68].

Statistical tests on SAM scores given by participants confirmed the a priori considerations about the different valence levels and the same intensity of the two smells chosen for the stimulation.

From the results of statistical analysis on HRV features, we observed that changes in ANS response, measured as differences in the values of the parameters in post and pre-stimulation sessions, were quantified especially from the LPP descriptors (14 out of 17 statistically significant features were extracted from LPP when we considered all the subjects). A significant variation of LF power parameter after the elicitation with the two odorants was reported in men (see Table 2). The HRV power spectral density in this frequency range is thought to be mixed sympathetic and parasympathetic activity, and this outcome confirmed the results obtained in [69], where a significant increase of the power in the MF band (from 0.08 to $0.15 \mathrm{~Hz}$ ) after emotional stimulation was recorded, studying short-term power spectrum analysis of HRV.

Gender differences had already been explored in the evaluation of physiological responses to olfactory stimulation [70-72]. Dijksterhuis et al. highlighted a more relevant
Table 5 Pattern recognition results obtained using different feature subsets for odorant discrimination

\begin{tabular}{llll}
\hline & All subjects & Men & Women \\
\hline Time & $61.67 \%$ & $66.67 \%$ & $73.33 \%$ \\
& RR mean & RR mean & RR mean \\
& RR std & RR std & \\
Frequency & $63.33 \%$ & $60.00 \%$ & $53.33 \%$ \\
& LF peak & LF peak & HF power \\
& HF peak & HF peak & LF/HF \\
& HF power & HF power & LF peak \\
& HFn & & $60.00 \%$ \\
LPP & $70.00 \%$ & $76.67 \%$ & $S(M=1)$ \\
& max $\left(M_{\mathrm{d}}\right)$ & auc $\left(S_{\mathrm{d}}\right)$ & \\
\hline
\end{tabular}

For each classification, the maximum of the accuracy and the corresponding most frequently selected features are reported 
lateralization of olfactory perception in men than in women, expressed in higher hedonic scores following odorous stimulation through the right nostril [71]. These differences, already hypothesized in the literature, have been confirmed in this study by the classification results. The results of the recognition accuracy increased when we split the dataset by gender and reached values of $83.33 \%$ and $73.33 \%$, with only three features, when we considered 35-s RR series from men and women, respectively. Interestingly, when we investigated which were the features most frequently selected by the RFE procedure among LPP descriptors and HRV features extracted in time and frequency domains, we found that two out of three were LPP descriptors. In order to compare the effectiveness of the three feature subsets in valence recognition, we used each subset as a separate input of the algorithm. We found that the maximum percentage of accuracy was reached using the LPP feature subset for both the groups of all subjects and men.

Our outcomes demonstrated that the hproposed LPP approach can be a promising tool for the assessment of odor hedonic tone perception, found to be strictly related to emotion regulation and pathological moods.

One limitation of this study is the number of subjects involved in the experimental protocol of olfactory stimulation that can lead to a low statistical power. For this reason, future works should consider a larger number of subjects.

The accuracy of the novel LPP approach could be applied to other affective computing experimental protocols, in order to define the relationship between the LPP descriptors and the ANS activity and to promote the implementation of this technique in all the fields which involve ultra-short time series (e.g., telemedicine and e-health monitoring).

Acknowledgments Work partially supported by the Italian Ministry of Education and Research (MIUR) in the framework of the CrossLab project (Departments of Excellence).

Funding information Research partly supported by AEI and FEDER under the projects RTI2018-097723-B-I00, by CIBER de Bioingenieria, Biomateriales y Nanomedicina through Instituto de Salud Carlos III, by LMP44-18 and BSICoS group (T39-17R) funded by Gobierno de Aragón.

\section{Compliance with ethical standards}

This study was approved by the Ethical Committee of the University of Pisa.

\section{References}

1. Chappell PB, Smith MA, Kilts CD, Bissette G, Ritchie J, Anderson C, Nemeroff CB (1986) Alterations in corticotropinreleasing factor-like immunoreactivity in discrete rat brain regions after acute and chronic stress. J Neurosci 6(10):2908-2914
2. Croy I, Symmank A, Schellong J, Hummel C, Gerber J, Joraschky P, Hummel T (2014) Olfaction as a marker for depression in humans. J Affect Disord 160(2014):80-86

3. Lombion-Pouthier S, Vandel P, Nezelof S, Haffen E, Millot J-L (2006) Odor perception in patients with mood disorders. J Affect Disord 90(2-3):187-191

4. Burón E, Bulbena A, Bulbena-Cabré A (2015) Olfactory functioning in panic disorder. J Affect Disord 175(2015):292-298

5. Oral E, Aydin M, Aydin N, Ozcan H, Hacimuftuoglu A, Sipal S, Demirci E (2013) How olfaction disorders can cause depression? The role of habenular degeneration. Neuroscience 240(2013):6369

6. Schiffman SS (1974) Physicochemical correlates of olfactory quality. Science (1974): 112-117

7. Igarashi M, Song C, Ikei H, Ohira T, Miyazaki Y (2014) Effect of olfactory stimulation by fresh rose flowers on autonomic nervous activity. J Alter Complement Med 20(9):727-731

8. Bensafi M, Rouby C, Farget V, Bertrand B, Vigouroux M, Holley A (2002) Autonomic nervous system responses to odours: the role of pleasantness and arousal. Chem Senses 27(8):703-709

9. Greco A, Nardelli M, Lanata A, Morelli M, Francesco FD, Scilingo E, Barbieri R, Valenza G (2017) Instantaneous assessment of hedonic olfactory perception using heartbeat nonlinear dynamics: a preliminary study. In: Computing in cardiology, 2017

10. Appelhans BM, Luecken LJ (2006) Heart rate variability as an index of regulated emotional responding. Rev Gen Psychol 10(3):229

11. Thayer JF, Hansen AL, Saus-Rose E, Johnsen BH (2009) Heart rate variability, prefrontal neural function, and cognitive performance: the neurovisceral integration perspective on selfregulation, adaptation, and health. Annals Behav Med 37(2):141153

12. Lane R, McRae K, Reiman E, Chen K, Ahern G, Thayer JF (2009) Neural correlates of heart rate variability during emotion. Neuroimage 44(1):213-222

13. Lane R, Reiman E, Ahern G, Thayer J (2001) 21. Activity in medial prefrontal cortex correlates with vagal component of heart rate variability during emotion. Brain Cogn 47(1-2):97-100

14. Leistedt SJ, Linkowski P, Lanquart JP, Mietus J, Davis RB, Goldberger AL, Costa MD (2011) Decreased neuroautonomic complexity in men during an acute major depressive episode: analysis of heart rate dynamics. Translational Psychiatry 1(7):e27

15. Valenza G, Nardelli M, Lanata A, Gentili C, Bertschy G, Kosel M, Scilingo E (2016) Predicting mood changes in bipolar disorder through heartbeat nonlinear dynamics. IEEE J Biomed Health Inform 20(4): 1034-1043

16. Valenza G, Nardelli M, Bertschy G, Lanata A, Scilingo E (2014) Mood states modulate complexity in heartbeat dynamics: a multiscale entropy analysis. EPL (Europhysics Letters) 107(1):18003

17. Nardelli M, Scilingo E, Valenza G (2019) Multichannel complexity index (MCI) for a multi-organ physiological complexity assessment, Physica A: Statistical Mechanics and its Applications: 121543

18. Valenza G, Lanata A, Scilingo E (2012) The role of nonlinear dynamics in affective valence and arousal recognition. IEEE Trans Affect Comput 3(2):237-249

19. Valenza G, Lanata A, Scilingo E (2012) Oscillations of heart rate and respiration synchronize during affective visual stimulation. IEEE Trans Inform Technol Biomed 16(4):683-690

20. Palomba D, Sarlo M, Angrilli A, Mini A, Stegagno L (2000) Cardiac responses associated with affective processing of unpleasant film stimuli. Int J Psychophysiology 36(1):45-57

21. Wiens S, Mezzacappa ES, Katkin ES (2000) Heartbeat detection and the experience of emotions. Cogn Emotion 14(3):417427 
22. Nardelli M, Valenza G, Greco A, Lanata A, Scilingo E (2015) Recognizing emotions induced by affective sounds through heart rate variability. IEEE Trans Affect Comput 6(4):385-394

23. Orini M, Bailón R, Enk R, Koelsch S, Mainardi L, Laguna P (2010) A method for continuously assessing the autonomic response to music-induced emotions through hrv analysis. Med Biol Eng Comput 48(5):423-433

24. Nardelli M, Greco A, Bianchi M, Scilingo E, Valenza G (2018) Classifying affective haptic stimuli through gender-specific heart rate variability nonlinear analysis. IEEE Transactions on Affective Computing

25. Nardelli M, Valenza G, Bianchi M, Greco A, Lanata A, Bicchi A, Scilingo E (2015) Gender-specific velocity recognition of caresslike stimuli through nonlinear analysis of heart rate variability. In: 2015 37th annual international conference of the IEEE engineering in medicine and biology society (EMBC). IEEE, 2015, pp 298-301

26. Takens F (1981) Detecting strange attractors in turbulence. In: Dynamical systems and turbulence, Warwick 1980, Springer, 1981, pp 366-381

27. Woo MA, Stevenson WG, Moser DK, Trelease RB, Harper RM (1992) Patterns of beat-to-beat heart rate variability in advanced heart failure. American Heart Journal 123(3):704-710

28. Woo MA, Stevenson WG, Moser DK, Middlekauff HR (1994) Complex heart rate variability and serum norepinephrine levels in patients with advanced heart failure. Journal of the American College of Cardiology 23(3):565-569

29. Hayano J, Takahashi H, Toriyama T, Mukai S, Okada A, Sakata S, Yamada A, Ohte N, Kawahara H (1999) Prognostic value of heart rate variability during long-term follow-up in chronic haemodialysis patients with end-stage renal disease. Nephrology Dialysis Transplantation 14(6):1480-1488

30. Tulppo MP, Makikallio T, Takala T, Seppanen T, Huikuri HV (1996) Quantitative beat-to-beat analysis of heart rate dynamics during exercise. American Journal of Physiology-Heart and Circulatory Physiology 271(1):H244-H252

31. Nardelli M, Greco A, Bolea J, Valenza G, Scilingo E, Bailon R (2017) Reliability of lagged poincaré plot parameters in ultra-short heart rate variability series: application on affective sounds. IEEE Journal of Biomedical and Health Informatics

32. Brennan M, Palaniswami M, Kamen P (2001) Do existing measures of poincare plot geometry reflect nonlinear features of heart rate variability? IEEE Trans Biomed Eng 48(11):1342-1347

33. Lerma C, Infante O, Pérez-Grovas H, José MV (2003) Poincaré plot indexes of heart rate variability capture dynamic adaptations after haemodialysis in chronic renal failure patients. Clinical Physiology and Functional Imaging 23(2):72-80

34. Koichubekov B, Riklefs V, Sorokina M, Korshukov I, Turgunova L, Laryushina Y, Bakirova R, Muldaeva G, Bekov E, Kultenova M (2017) Informative nature and nonlinearity of lagged poincaré plots indices in analysis of heart rate variability. Entropy 19(10):523

35. Nardelli M, Greco A, Bolea J, Valenza G, Scilingo E, Bailón R (2017) Investigation of lagged poincaré plot reliability in ultrashort synthetic and experimental heart rate variability series. In: 2017 IEEE 39th annual international conference of the engineering in medicine and biology society (EMBC). IEEE, 2017

36. Khandoker A, Karmakar C, Brennan M, Palaniswami M, Voss A (2013) Poincaré plot methods for heart rate variability analysis. Springer, 2013

37. Seeck A, Baumert M, Fischer C, Khandoker A, Faber R, Voss A (2011) Advanced poincaré plot analysis differentiates between hypertensive pregnancy disorders. Physiological Measurement 32(10): 1611
38. Voss A, Fischer C, Schroeder R, Figulla H, Goernig M et al (2010) Segmented poincare plot analysis for risk stratification in patients with dilated cardiomyopathy. Methods of Information in Medicine 49(5):511-515

39. Voss A, Fischer C, Schroeder R, Figulla HR, Goernig M (2012) Lagged segmented poincaré plot analysis for risk stratification in patients with dilated cardiomyopathy. Med Biol Eng Comput 50(7):727-736

40. Nardelli M, Greco A, Valenza G, Lanata A, Bailón R, Scilingo E (2017) A novel heart rate variability analysis using lagged poincaré plot: A study on hedonic visual elicitation. In: 2017 39th annual international conference of the IEEE engineering in medicine and biology society (EMBC). IEEE, 2017, pp 23002303

41. Nardelli M, Greco A, Danzi O, Perlini C, Tedeschi F, Scilingo E, Del Piccolo L, Valenza G (2019) Cardiovascular assessment of supportive doctor-patient communication using multi-scale and multi-lag analysis of heartbeat dynamics. Med Biol Eng Comput 57(1):123-134

42. Yousem DM, Maldjian JA, Siddiqi F, Hummel T, Alsop DC, Geckle RJ, Bilker WB, Doty RL (1999) Gender effects on odorstimulated functional magnetic resonance imaging. Brain Res 818(2):480-487

43. Guzik P, Piskorski J, Krauze T, Schneider R, Wesseling KH, Wykretowicz A, Wysocki H (2007) Correlations between the poincare plot and conventional heart rate variability parameters assessed during paced breathing. J Physiological Sci 57(1):63-71

44. Piskorski J, Guzik P (2005) Filtering poincare plots. Comput Methods Sci Technol 11(1):39-48

45. Mateo J, Laguna $P$ (2000) Improved heart rate variability signal analysis from the beat occurrence times according to the ipfm model. IEEE Trans Biomed Eng 47(8):985-996

46. Orini M, Bailón R, Mainardi L, Laguna P (2012) Synthesis of hrv signals characterized by predetermined time-frequency structure by means of time-varying arma models. Biomed Signal Process Control 7(2):141-150

47. Schäfer D, Nil M, Herzig D, Eser P, Saner H, Wilhelm M (2015) Good reproducibility of heart rate variability after orthostatic challenge in patients with a history of acute coronary syndrome. $\mathrm{J}$ Electrocardiology 48(4):696-702

48. Guzzetti S, La Rovere MT, Pinna GD, Maestri R, Borroni E, Porta A, Mortara A, Malliani A (2005) Different spectral components of $24 \mathrm{~h}$ heart rate variability are related to different modes of death in chronic heart failure. European Heart Journal 26(4):357-362

49. Naudin M, El-Hage W, Gomes M, Gaillard P, Belzung C, Atanasova B (2012) State and trait olfactory markers of major depression. PLoS One 7(10):e46938

50. Greco A, Lanata A, Valenza G, Di Francesco F, Scilingo E (2016) Gender-specific automatic valence recognition of affective olfactory stimulation through the analysis of the electrodermal activity. In: 2016 IEEE 38th annual international conference of the engineering in medicine and biology society (EMBC). IEEE, 2016, pp 399-402

51. Russell JA (1980) A circumplex model of affect. Journal of Personality and Social Psychology 39(6):1161

52. Bradley MM, Lang PJ (1994) Measuring emotion: the selfassessment manikin and the semantic differential. Journal of Behavior Therapy and Experimental Psychiatry 25(1):49-59

53. Pan J, Tompkins WJ (1985) A real-time qrs detection algorithm. IEEE Transactions on Biomedical Engineering (3):230-236

54. Tarvainen MP, Niskanen J-P, Lipponen JA, Ranta-Aho PO, Karjalainen PA (2014) Kubios hrv-heart rate variability analysis software. Computer Methods and Programs in Biomedicine 113(1):210-220 
55. Mateo J, Laguna P (2003) Analysis of heart rate variability in the presence of ectopic beats using the heart timing signal. IEEE Transactions on Biomedical Engineering 50(3):334-343

56. Duda RO, Hart PE, Stork DG (2012) Pattern classification. Wiley, New York, p 2012

57. Esterman M, Tamber-Rosenau BJ, Chiu Y-C, Yantis S (2010) Avoiding non-independence in fmri data analysis: leave one subject out. Neuroimage 50(2):572-576

58. Yan K, Zhang D (2015) Feature selection and analysis on correlated gas sensor data with recursive feature elimination. Sensors Actuators B Chem 212(2015):353-363

59. Nardelli M, Valenza G, Cristea IA, Gentili C, Cotet C, David D, Lanata A, Scilingo E (2015) Characterizing psychological dimensions in non-pathological subjects through autonomic nervous system dynamics. Frontiers in Computational Neuroscience 9

60. McNames J, Aboy M (2006) Reliability and accuracy of heart rate variability metrics versus ecg segment duration. Medical and Biological Engineering and Computing 44(9):747-756

61. Nardelli M, Greco A, Bolea J, Valenza G, Scilingo E, Bailón R (2018) Reliability of lagged poincaré plot parameters in ultrashort heart rate variability series: application on affective sounds. IEEE Journal of Biomedical and Health Informatics 22(3):741-749

62. Alaoui-Ismaili O, Vernet-Maury E, Dittmar A, Delhomme G, Chanel J (1997) Odor hedonics: connection with emotional response estimated by autonomic parameters. Chemical Senses 22(3):237-248

63. Bensafi M, Sobel N, Khan RM (2007) Hedonic-specific activity in piriform cortex during odor imagery mimics that during odor perception. Journal of Neurophysiology 98(6):3254-3262

64. Thuerauf N, Gossler A, Lunkenheimer J, Lunkenheimer B, Maihöfner C, Bleich S, Kornhuber J, Markovic K, Reulbach U (2008) Olfactory lateralization: odor intensity but not the hedonic estimation is lateralized. Neurosci Lett 438(2):228-232

65. Cain D, Bindra D (1972) Responses of amygdala single units to odors in the rat. Experimental Neurology 35(1):98-110

66. Zald DH, Pardo JV (1997) Emotion, olfaction, and the human amygdala: amygdala activation during aversive olfactory stimulation. Proceedings of the National Academy of Sciences 94(8):4119-4124

67. Plum F (1960) Handbook of physiology. AMA Archives of Neurology 2(3):360-360

68. Van Toller S (1988) Emotion and the brain. In: Perfumery, Springer, 1988, pp 121-143

69. McCraty R, Atkinson M, Tiller WA, Rein G, Watkins AD (1995) The effects of emotions on short-term power spectrum analysis of heart rate variability. The American Journal of Cardiology 76(14):1089-1093

70. Wysocki CJ, Gilbert AN (1989) National geographic smell survey: effects of age are heterogenous. Annals of the New York Academy of Sciences 561(1):12-28

71. Dijksterhuis GB, Møller P, Bredie WL, Rasmussen G, Martens M (2002) Gender and handedness effects on hedonicity of laterally presented odours. Brain Cogn 50(2):272-281

72. Thuerauf N, Reulbach U, Lunkenheimer J, Lunkenheimer B, Spannenberger R, Gossler A, Maihöfner C, Bleich S, Kornhuber J, Markovic K (2009) Emotional reactivity to odors: olfactory sensitivity and the span of emotional evaluation separate the genders. Neurosci Lett 456(2):74-79

73. Tarvainen MP, Ranta-Aho PO, Karjalainen PA (2002) An advanced detrending method with application to hrv analysis. IEEE Trans Biomed Eng 49(2):172-175
74. Julian LJ (2011) Measures of anxiety: state-trait anxiety inventory (stai), beck anxiety inventory (bai), and hospital anxiety and depression scale-anxiety (hads-a). Arthritis Care \& Research 63(S11):S467-S472

75. Watson D, Clark LA, Tellegen A (1988) Development and validation of brief measures of positive and negative affect: the panas scales. Journal of Personality and Social Psychology 54(6): 1063

76. Carrasco RGOYS, Gaitán MJ (2001) Correlation among poincare plot indexes and time and frequency domain measures of heart rate variability. J Med Eng Technol 25(6):240-248

Publisher's note Springer Nature remains neutral with regard to jurisdictional claims in published maps and institutional affiliations.

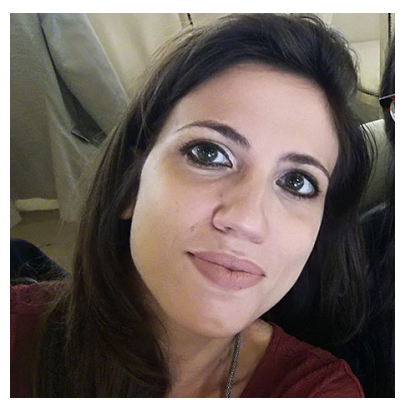

M. Nardelli received a Ph.D. in Information Engineering from the University of Pisa (2017). Her research is mainly related to the study of nonlinear dynamics in biosignals of central and autonomic nervous systems.

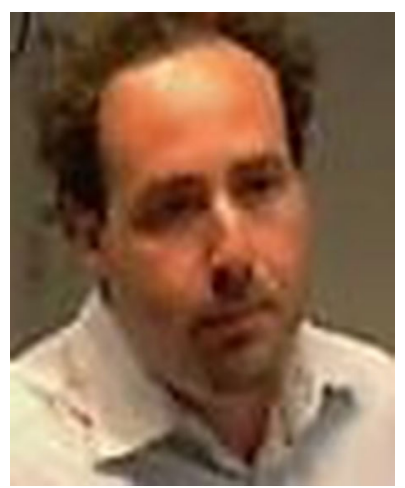

G. Valenza Ph.D. in Automation, Robotics, and Bioengineering, is currently an Assistant Professor of Bioengineering at the University of Pisa.

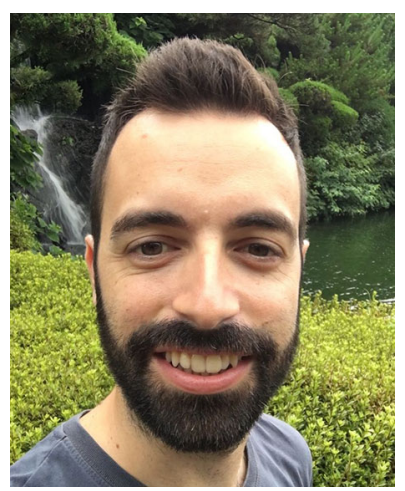

A. Greco received the Ph.D. in Automatics, Robotics, and Bioengineering from the University of Pisa (2015). His main research interests are physiological modeling and biomedical signal processing. 


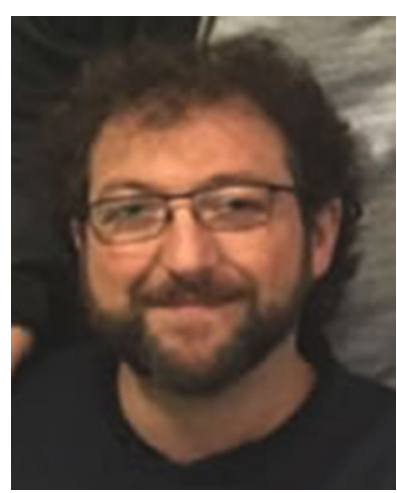

A. Lanatá received the $\mathrm{Ph} . \mathrm{D}$. degree in Automation, Robotics, and Bioengineering at University of Pisa (2006). His main research interests are the development of wearable systems and smartphonebased apps for mental and physical wellbeing.

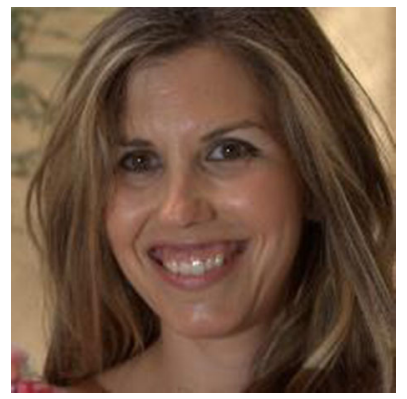

R. Bailón is an Associate Professor in the Department of Electronic Engineering and Communications, University of Zaragoza (UZ), and Researcher with the I3A and CIBER-BBN, Spain.

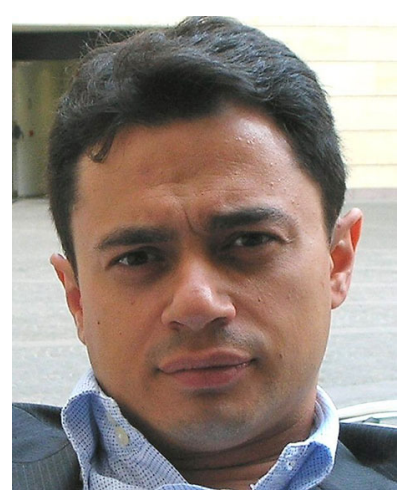

E. P. Scilingo Ph.D., is an Associate Professor in Electronic and Information Bioengineering at the University of Pisa. 